\title{
Four-wave mixing mediated by the capture of electrons and holes in semiconductor quantum-well laser amplifiers
}

\author{
Roberto Paiella, Guido Hunziker, and Kerry J. Vahala \\ Department of Applied Physics, Mail Stop 128-95, California Institute of Technology, Pasadena, \\ California 91125 \\ Uzi Koren \\ Bell Laboratories, Lucent Technologies, Holmdel, New Jersey 07733
}

(Received 18 June 1997; accepted for publication 18 October 1997)

\begin{abstract}
An experimental technique based on frequency-resolved four-wave mixing is proposed for the investigation of phonon-assisted capture of electrons and holes in electrically pumped semiconductor quantum wells. We show how this technique can be used to directly measure the intrinsic capture lifetime, with no need for involved numerical fits. We also present experimental results from an application of the technique to a multiquantum-well semiconductor optical amplifier. The possible impact of phase matching on the results is discussed. (C) 1997 American Institute of Physics. [S0003-6951(97)00351-3]
\end{abstract}

The electrical carriers injected to the barrier region of a semiconductor quantum-well structure are captured in the quantum-well states with a finite characteristic rate, the inverse capture lifetime $1 / \tau_{\text {cap }}$. Such capture processes (effectively intersubband transitions between 3D states delocalized across the barrier region and quantum-confined 2D states in the quantum well) have attracted considerable attention in recent years, ${ }^{1-8}$ within the broad context of ultrafast spectroscopy of semiconductor microstructures. ${ }^{1}$ Furthermore, they are of significant interest to the semiconductor laser community, because of their direct relevance to the dynamic and spectral features of quantum-well lasers. ${ }^{4-7}$ It is generally agreed that the capture of electrons and holes in polar semiconductors mainly occurs through the emission of optical phonons, although carrier-carrier scattering also plays a role $^{7}$ in the presence of the large carrier densities typical of laser operation. In any case, the direct measurement of the "intrinsic" capture lifetime is complicated, because experiments typically measure a compound response including, e.g., drift and diffusion across the barrier region, or energy relaxation within the $3 \mathrm{D}$ continuum and within the $2 \mathrm{D}$ subbands.

Recently, nondegenerate four-wave mixing (FWM) has emerged as a useful frequency-domain technique for the direct measurement of the ultrafast lifetimes governing relaxation processes in excited semiconductors. ${ }^{9-12}$ In this letter, we present a novel experimental technique based on frequency-resolved FWM to directly study capture processes in (multi)quantum-well semiconductor optical amplifiers (SOAs). In the experiment, a small-signal carrier density modulation is generated in the electronic states near the barrier band-edges (by photomixing of two pump waves), and is then probed in the quantum wells as a function of the modulation frequency. A similar technique has been used to investigate the capture dynamics in a quantum-well laser in Ref. 6, where the carrier density modulation was produced by injection of a directly modulated beam from another semiconductor laser. In that work, the modulation frequency was limited by the bandwidth of the laser source and the detection electronics to around $20 \mathrm{GHz}$. The use of FWM, as in the experiment described here, allows us to extend the measured bandwidth to a few hundreds of $\mathrm{GHz}$, i.e., well beyond the typical values for the intrinsic capture rate, so that in principle it can be measured directly, with no need for involved theoretical fits. On the other hand, phase-matching must be included in the interpretation of the experimental data.

A pictorial description of the experimental configuration is given in Fig. 1. The structure studied is a multiquantumwell InGaAs/InGaAsP SOA consisting of three pairs of a $160 \AA$ tensile and a $75 \AA$ compressively-strained quantum well, separated by $100 \AA$ barriers. ${ }^{13}$ Three cw waves, generated by temperature-tunable distributed-feedback lasers, are coupled into the SOA waveguide: two pump waves with frequencies matching the band gap of the InGaAsP barriers (nominally $0.97 \mathrm{eV}$ ), ${ }^{14}$ and one probe wave with frequency near the gain peak of the SOA (in the $0.8 \mathrm{eV}$ band). Beating of the two pump waves generates a modulation of the carrier density in the barrier region, at their difference frequency $\Omega$. A fraction of this modulation is captured in the quantum wells where it then acts to scatter energy from the probe laser

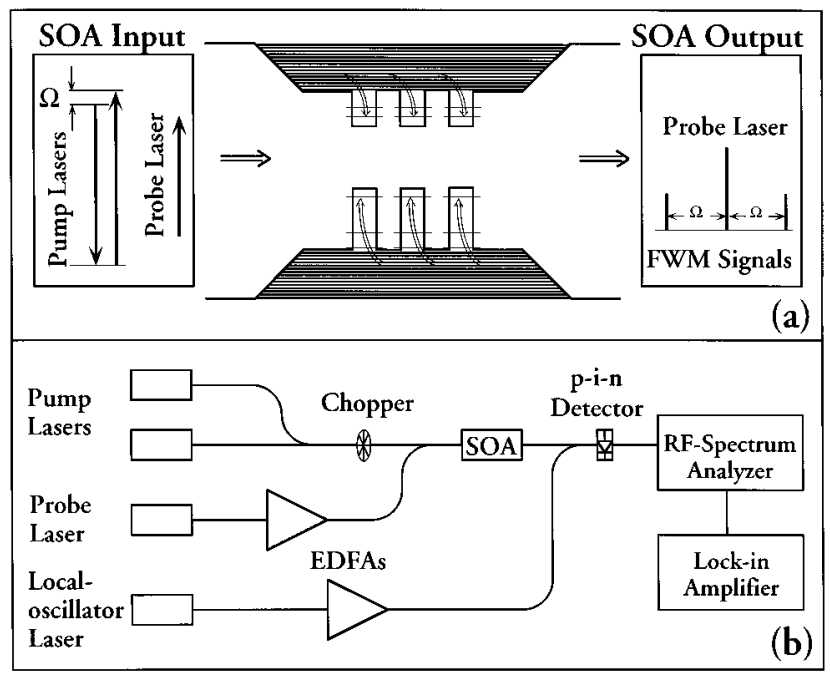

FIG. 1. (a) Pictorial description of the experiment, and (b) schematics of the experimental layout. 


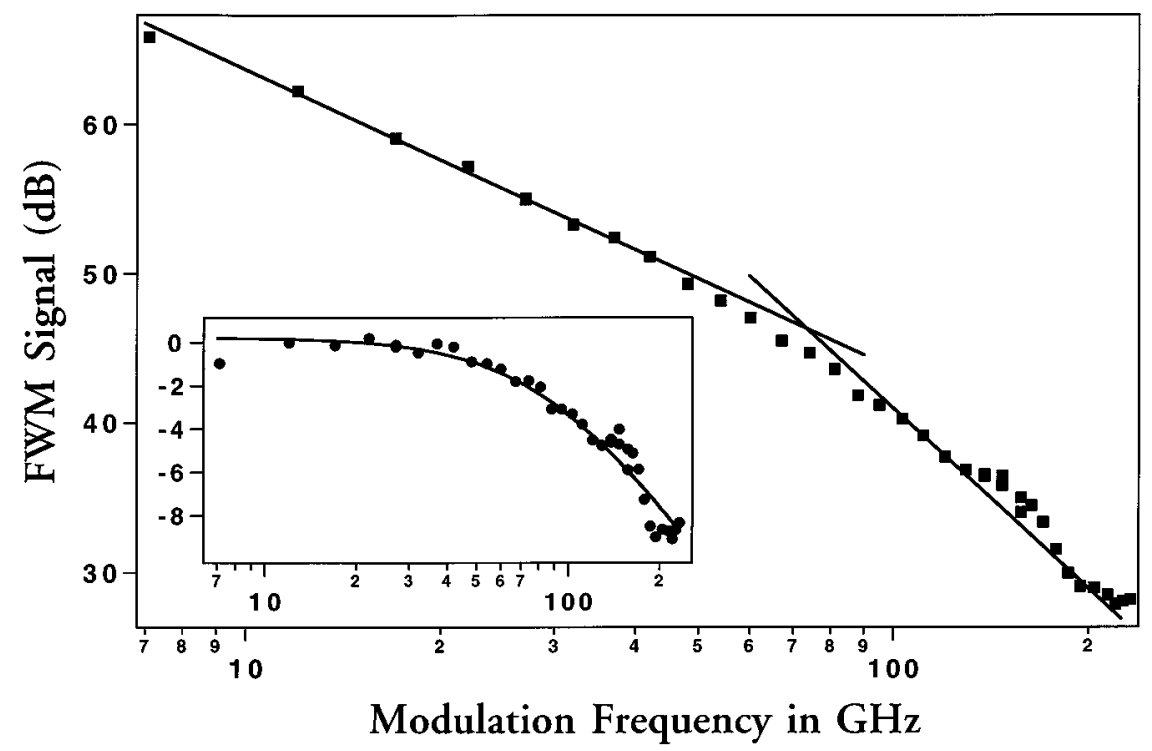

FIG. 2. Measured optical intensity of the FWM signal versus modulation frequency (the origin of the vertical axis is arbitrary). As emphasized by the continuous lines, the slope of the data changes from -20 to $-40 \mathrm{~dB} / \mathrm{dec}$ with increasing modulation frequency. The inset displays the same data with the initial $20 \mathrm{~dB} / \mathrm{dec}$ roll-off subtracted; the continuous curve is a single-pole frequency response with pole at $88 \mathrm{GHz}=1 /(2 \pi \times 1.8 \mathrm{psec})$.

beam into two FWM sidebands, as shown in Fig. 1(a). Information on the carrier capture can then be inferred from the relative intensity of either FWM signal as a function of the modulation frequency $\Omega$. In particular, we expect the FWM conversion efficiency to become negligibly small at modulation frequencies larger than the capture rate. At these frequencies, the probability of the modulated carrier distribution being captured in the quantum wells within a few modulation periods becomes negligibly small. Furthermore, due to the close proximity of the frequencies of the pump waves to the barrier band gap, the limiting rate is most likely that of the intrinsic capture, fully isolated from other transport effects.

The physical layout of the experiment is shown in Fig. 1(b). The SOA (780 $\mu \mathrm{m}$ long) was biased with a dc current of $100 \mathrm{~mA}$. Approximately $2 \mathrm{~mW}$ of pump power and 17 $\mathrm{mW}$ of probe power (after preamplification in a high-gain Erbium-doped fiber amplifier) were coupled into the active waveguide. The probe wave is linearly polarized along the TM axis of the SOA waveguide, so that only the tensile wells are probed (compressively-strained quantum wells have negligible gain for TM light); this avoids any complication arising from possibly having different capture rates in the two types of wells. The SOA output was combined with a tunable optical local oscillator, and the beat signal between the local oscillator and either FWM sideband was detected at a constant frequency in a radio-frequency spectrum analyzer. In order to increase the signal-to-noise ratio, we fed the ana$\log$ output of the spectrum analyzer to a lock-in amplifier (locked to a chopper placed in the optical path of the pump waves), where the signal was measured.

A typical trace is shown in Fig. 2, which refers to the frequency-upconverted FWM sideband with the modulation frequency ranging from 7 to $230 \mathrm{GHz}$. As emphasized by the continuous lines, the data exhibit a $20 \mathrm{~dB} / \mathrm{dec}$ roll-off at low modulation frequencies and a $40 \mathrm{~dB} / \mathrm{dec}$ roll-off at modulation frequencies exceeding about $90 \mathrm{GHz}$. The former roll- off is associated with the interband stimulated recombination of the carriers in the quantum wells (occurring at a rate $1 /\left(2 \pi \tau_{s}\right)$ of approximately $1.2 \mathrm{GHz}$ under the present experimental condition ${ }^{11}$ ). Since the emphasis here is on the features related to the capture process, this constant roll-off was normalized out (notice that the exact value of $1 /\left(2 \pi \tau_{s}\right)$ used in this normalization is irrelevant, provided it is smaller than the smallest modulation frequency measured). The result is shown in the inset of Fig. 2, which clearly suggests the presence of another pole at $88 \mathrm{GHz}$ (corresponding to a lifetime of $1.8 \mathrm{psec}$ ). Additional structures are observed at larger modulation frequencies. The same features were observed in the frequency-downconverted data.

A two-pole frequency response is consistent with a simple model of the capture dynamics based on the following set of rate equations (in the frequency domain)

$$
\begin{aligned}
& -i \Omega N_{2 \mathrm{D}}^{(\Omega)}=-\left(\frac{1}{\tau_{s}}+\frac{1}{\tau_{\mathrm{esc}}}\right) N_{2 \mathrm{D}}^{(\Omega)}+\frac{N_{3 \mathrm{D}}^{(\Omega)}}{\tau_{\text {cap }}}, \\
& -i \Omega N_{3 \mathrm{D}}^{(\Omega)}=-\left(\frac{1}{\tau_{s}}+\frac{1}{\tau_{\mathrm{cap}}}\right) N_{3 \mathrm{D}}^{(\Omega)}+\frac{N_{2 \mathrm{D}}^{(\Omega)}}{\tau_{\mathrm{esc}}}+g_{3 \mathrm{D}}^{(\Omega)} .
\end{aligned}
$$

Here $N_{2 \mathrm{D}}^{(\Omega)}$ denotes the small-signal density of carriers confined inside each (tensile) well; $N_{3 \mathrm{D}}^{(\Omega)}$ is the density of unbound carriers involved in the capture/escape processes with the same quantum well (and directly modulated by the beating of the pump waves, at a rate $g_{3 \mathrm{D}}^{(\Omega)}$ ); and $\tau_{\text {cap }}$ and $\tau_{\text {esc }}$ are the intrinsic capture and escape lifetimes. Notice that we are not including in this model any effect related to transport in real space, such as diffusion or drift, as appropriate for excitation near the barriers band-edges. ${ }^{15}$ Furthermore, we are taking the capture and escape rates to be constants, independent of the excitation conditions (for instance, they would depend on $\Omega$ if the occupancy of the phonon modes involved in the capture/escape processes were significantly perturbed from equilibrium by the capture dynamics itself). Finally, we 
do not explicitly consider the capture of both conductionband electrons and holes, since the frequency response measured in this experiment should be dominated by the slower carrier type (presumably the electrons ${ }^{3}$ ).

The field amplitude of the FWM signal (as a function of $\Omega$ ) is proportional to the amplitude of the carrier density modulation that generates it, $N_{2 \mathrm{D}}^{(\Omega)}$. Solving the coupled set of Eqs. (1), one finds

$$
N_{2 \mathrm{D}}^{(\Omega)}=\frac{1 / \tau_{\text {cap }}}{\left(-i \Omega+1 / \tau_{s}\right)\left(-i \Omega+1 / \tau_{\text {cap }}+1 / \tau_{\text {esc }}+1 / \tau_{s}\right)} g_{3 \mathrm{D}}^{(\Omega)},
$$

so that on the basis of this simple model we expect the FWM conversion efficiency to exhibit one pole at $1 / \tau_{s}$ and another at $1 / \tau_{\text {cap }}+1 / \tau_{\text {esc }}+1 / \tau_{s}\left(\approx 1 / \tau_{\text {cap }}\right.$ since in general the capture lifetime is shorter than both the stimulated recombination and the escape lifetimes $\left.{ }^{5,10}\right)$. As a result, $\tau_{\text {cap }}$ can be directly read off the experimental data, provided that the frequency dependence is entirely ascribed to the carrier-density modulation response described by Eq. (2). If we assume that this is indeed the case for the data of Fig. 2, we immediately obtain an electronic capture lifetime of 1.8 psec. Incidentally, this estimate is consistent with a recent measurement of the interwell transport rate in the same SOA,${ }^{10}$ from which a value of 1.5 psec for $\tau_{\text {cap }}$ (and 8 psec for $\tau_{\text {esc }}$ ) was inferred.

However, it is obvious from Fig. 2 that the two-pole frequency response of Eq. (2) is inadequate to fit the data at the higher measured modulation frequencies. In particular, notice the two shoulders in the data around 140 and 200 $\mathrm{GHz}$, which resemble the first two nulls of a phase-matching frequency response. Due to the large frequency difference between the pump waves on the one hand, and the probe and FWM signal on the other, the wave number mismatch $\Delta k$ entering the FWM conversion efficiency may be large enough to produce zeroes at these detuning frequencies. However, the observed pronounced features can be fit to a damped phase-matching frequency response only if the damping (i.e., the total absorption of the pump waves in the SOA) is smaller than approximately $10 \mathrm{~dB}$, whereas a loss of more than $20 \mathrm{~dB}$ was actually measured. An alternative explanation for the high-frequency features of the data of Fig. 2 (based on a resonant coupling between the carrier capture dynamics and that of the phonon modes involved in the capture process) is under consideration. In order to resolve this issue, further experimental work is required; in particular to test for the presence of phase-matching. This could be accomplished by using pump waves of shorter wavelength or SOA active layers having lower quantum-well barrier energies, so as to increase pump absorption and hence decrease the effective interaction length. Unfortunately, these measurements are not possible with our existing setup.

The ambiguities just discussed regarding the role of phase-matching prevent us from making a definite statement on the magnitude of $\tau_{\text {cap }}$. In particular, if the high-frequency features in the data are due to phase-matching effects, these same effects could be responsible for most of the roll-off observed in the normalized data (inset of Fig. 2). Then the above mentioned estimate of $1 /(1.8 \mathrm{psec})$ should be regarded as a lower limit to the capture rate under the present experimental condition (which may produce a particularly fast cap- ture rate as a result of modulating states at the barrier edges ${ }^{14}$ ). Furthermore, at the higher measured modulation frequencies the temperature dynamics of the captured carrier distributions may also affect the frequency response. In any case, the results presented here clearly indicate how the proposed technique can be used to measure the strength of the carrier-density modulation captured in the well, up to modulation frequencies larger than the expected intrinsic capture rate. This feature makes the technique particularly attractive, because (provided that phase-matching can be eliminated as a source of frequency dependence) the capture lifetime can then be directly read off the data, after the simple normalization carried out in the inset of Fig. 2.

The authors thank Dr. N. Kwong and Dr. T. Schrans, ORTEL Corp., for the loan of the DFB lasers used in the experiment. This work was supported by ARPA (Contract No. DAAL 01-94-K-03430) and the National Science Foundation (Grant No. ECS-9412862).

${ }^{1}$ For an up-to-date review of nonlinear ultrafast spectroscopy of semiconductors, see J. Shah, Ultrafast Spectroscopy of Semiconductors and Semiconductor Nanostructures (Springer, Berlin, Germany, 1996).

${ }^{2}$ B. K. Ridley. Rep. Prog. Phys. 54, 169 (1991).

${ }^{3}$ B. Deveaud, J. Shah, T. C. Damen, and W. T. Tsang, Appl. Phys. Lett. 52, 1886 (1988).

${ }^{4}$ S. Weiss, J. M. Wiesenfeld, D. S. Chemla, G. Raybon, G. Sucha, M. Wegener, G. Eisenstein, C. A. Burrus, A. G. Dentai, U. Koren, B. I. Miller, H. Temkin, R. A. Logan, and T. Tanbun-Ek, Appl. Phys. Lett. 60, 9 (1992).

${ }^{5}$ S. C. Kan, D. Vassilovski, T. C. Wu, and K. Y. Lau, Appl. Phys. Lett. 61, 752 (1992).

${ }^{6}$ D. Vassilovski, T. C. Wu, S. Kan, K. Y. Lau, and C. E. Zah, IEEE Photonics Technol. Lett. 7, 706 (1995)

${ }^{7}$ J. E. M. Haverkort, P. W. M. Blom, P. J. van Hall, J. Claes, and J. H. Wolter, Phys. Status Solidi A 188, 139 (1995).

${ }^{8}$ G. Lenz, E. P. Ippen, J. M. Wiesenfeld, M. A. Newkirk, and U. Koren, Appl. Phys. Lett. 68, 2933 (1996).

${ }^{9}$ J. Zhou, N. Park, J. W. Dawson, K. J. Vahala, M. A. Newkirk, and B. I. Miller, Appl. Phys. Lett. 63, 1179 (1993).

${ }^{10}$ R. Paiella, G. Hunziker, K. J. Vahala, and U. Koren, Appl. Phys. Lett. 69, 4142 (1996).

${ }^{11}$ G. Hunziker, R. Paiella, K. J. Vahala, and U. Koren, IEEE Photonics Technol. Lett. 9, 907 (1997).

${ }^{12}$ R. Paiella, G. Hunziker, U. Koren, and K. J. Vahala, IEEE J. Sel. Top. Quantum Electron. 3, 529 (1997).

${ }^{13}$ M. A. Newkirk, B. I. Miller, U. Koren, M. G. Young, M. Chen, R. M. Jopson, and C. A. Burrus, IEEE Photonics Technol. Lett. 4, 406 (1993).

${ }^{14}$ The photon energy of the pump waves was $0.94 \mathrm{eV}$, which is smaller than the barrier band gap by an amount on the order of the thermal energy $k T$. Accounting for thermal broadening, we conclude that the electronic states directly modulated by these waves are not bound in any quantum well (i.e., they are purely 3D states), although their wavefunctions are somewhat localized near the wells. As a result, capture from these states is probably faster than capture from more plane-wave-like states that are higher in energy. The study of the dependence of the capture rate on the excitation energy will be the subject of a future work.

${ }^{15}$ In cases where carrier diffusion cannot be neglected, an analysis based on the model of interwell carrier dynamics described in Ref. 12 shows that Eq. (2) remains valid with the intrinsic capture rate rescaled by a geometrical factor (approximately the ratio of the quantum-wells volume to the barriers volume, which in the present structure is close to unity anyway). This is consistent with the results of Refs. 5 and 6. 